\title{
Effects of solvent and temperature on regioregularity of poly(3-hexylthiophene-2,5-diyl) prepared by chemical oxidative polymerization
}

\author{
Hiroki Fukumoto, Yoshinobu Omori and Takakazu Yamamoto \\ Polymer Journal (2013) 45, 462-465; doi:10.1038/pj.2012.152; published online 8 August 2012
}

Keywords: chemical oxidative polymerization; $\pi$-conjugated polymer; poly(3-hexylthiophene-2,5-diyl); regioregularity; solvent effect; stacking

\section{INTRODUCTION}

$\pi$-Conjugated polymers bearing electronic and optical functionalities are the subject of many papers. Control of regioregularity of $\pi$ conjugated polymers is important to improve their electronic and optical properties. ${ }^{1-4}$ For example, regioregular head-to-tail poly (3-alkylthiophene-2,5-diyl), HT-P3RTh, shows a higher electrical conductivity than the corresponding regio-random poly (3-alkylthiophene-2,5-diyl), rand-P3RTh. ${ }^{5}$

Regioregular $\pi$-conjugated polymers are usually obtained by organometallic polycondensation ${ }^{5-7}$ (OMP) (for example, equation (1)), and OMP gives HT-P3RTh with a HT content higher than $98 \%{ }^{5,6}$<smiles>[R19]c1cc([R])c(Br)s1</smiles>

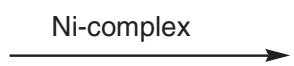
$M=M g, Z n$<smiles>[R]c1cc(-c2sc(-c3sc(-c4sc(C)cc4[R])cc3[R])cc2[R])sc1C</smiles>

On the other hand, chemical oxidative polymerization ${ }^{8-10}$ (COP) of 3-alkylthiophene proceeds in a simpler reaction system, using more common reagents such as $\mathrm{FeCl}_{3}$.
Typical COP using $\mathrm{FeCl}_{3}$ has been carried out mostly in halogenated solvents, such as $\mathrm{CHCl}_{3}$ at room temperature (equation (2)).
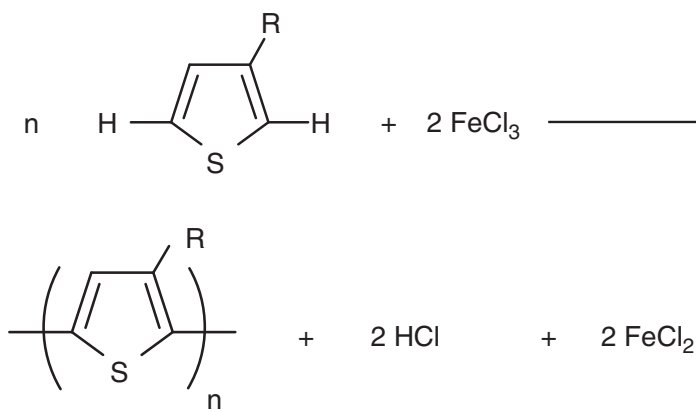

\section{P3RTh}

Effects of reaction conditions of COP have been studied, and Ueda et al. ${ }^{11-13}$ have revealed that the HT content of P3RTh can be increased when polymerization is carried out in $\mathrm{CHCl}_{3}$ at low temperature, for example, at $-45^{\circ} \mathrm{C} .{ }^{11}$ They optimized reaction conditions, such as monomer concentration and reaction time, and obtained P3RTh with an HT content of $88 \%$. However, solvent effect on the regioregularity of P3RTh was not revealed. Revealing effects of regioregularity of the COP-prepared P3RTh on its physical and chemical properties is considered to be interesting; physical and chemical properties of P3RTh have mainly been studied with OMPprepared P3RTh.

Here we report solvent and temperature effects on regioregularity and polydispersity (PDI) of the COP-prepared P3HexTh ( $\mathrm{R}$ in $\mathrm{P} 3 \mathrm{RTh}=\mathrm{hexyl}$ ) as well as effects of the regioregularity of the COP-prepared P3HexTh on its physical and chemical properties. 


\section{EXPERIMENTAL PROCEDURE}

\section{Measurements}

NMR spectra and IR spectra were recorded on a JEOL EX-300 and JASCO IR800 spectrometers, respectively. UV-vis spectra of a polymer solution and film were measured with a Shimadzu (Kyoto, Japan) UV-3100PC and UV-2500PC spectrometers. Gel-permeation chromatography was carried out with a Shimadzu SIL-20A liquid chromatograph using chloroform as the eluent (polystyrene standards). Powder X-ray diffraction (XRD) patterns were obtained by using a Rigaku (Tokyo, Japan) RINT2100 powder X-ray diffractometer with $\mathrm{CuK} \alpha(1.54 \AA)$ radiation. Electrical conductivity of the p-doped polymer film was measured with a LORESTA-IP MCP-T250 resistometry by a four-probe method.

\section{Materials}

3-Hexylthiophene was purchased from Tokyo Chemical Industry Co., Ltd. (Tokyo, Japan) Anhydrous organic solvents were purchased from Kanto Chemical Co., Inc. (Tokyo, Japan) and stored under $\mathrm{N}_{2}$ before use.

\section{Preparation of $\mathrm{P} 3 \mathrm{HexTh}$}

A typical example of the preparation of $\mathrm{P} 3 \mathrm{HexTh}$ : 3-Hexylthiophene $(0.50 \mathrm{~g}$, $3.0 \mathrm{mmol}$ ) and $\mathrm{FeCl}_{3}(1.94 \mathrm{~g}, 12.0 \mathrm{mmol})$ were added to anhydrous chloroform $(50 \mathrm{ml})$ at $-60{ }^{\circ} \mathrm{C}$ in a Schlenk tube under $\mathrm{N}_{2} .{ }^{8}$ After stirring at the temperature for $24 \mathrm{~h}$ under $\mathrm{N}_{2}$, the reaction mixture was poured into methanol, and the precipitate was collected by filtration. The crude product dissolved in chloroform was de-doped by treatment with an aqueous solution of hydrazine. The organic layer was separated and the solvent was removed by evaporation to give $\mathrm{P} 3 \mathrm{HexTh}$ as a green film. The polymer was extracted with a Soxhlet extractor with chloroform and the chloroform solution was added to methanol to recover purified P3HexTh. Purified P3HexTh was collected by filtration and dried under reduced pressure to give a green solid (Run 6 in Table 1) $(0.37 \mathrm{~g}, 74 \%$ yield $) .{ }^{1} \mathrm{H}$ NMR $\left(\mathrm{CDCl}_{3}\right): \delta=6.98(\mathrm{~s}, 1 \mathrm{H} . \mathrm{H}$ at the thiophene ring), $2.80\left(\mathrm{t}, 1.74 \mathrm{H}, \alpha-\mathrm{CH}_{2}\right.$, HT unit), $2.55\left(\mathrm{t}, 0.26 \mathrm{H}, \alpha-\mathrm{CH}_{2}, \mathrm{HH}\right.$ unit), 1.77 (quintet, $\left.2 \mathrm{H}, \beta-\mathrm{CH}_{2}\right), 1.50-1.30\left(\mathrm{~m}, 6 \mathrm{H},-\left(\mathrm{CH}_{2}\right)_{3}-\right), 0.92(\mathrm{t}, 3 \mathrm{H}$, $-\mathrm{CH}_{3}$ ). IR (KBr): 2952, 2905, 2851, 1750, 1643, 1524, 1452, 1393, 1363, 1226, $1143,1071,923,845,821,762,679,655,619 \mathrm{~cm}^{-1}$. Anal. calcd for $\left(\mathrm{C}_{10} \mathrm{H}_{14} \mathrm{~S}\right)_{n}$ : C, 72.23; H, 8.49; S, 19.28\%. Found: C, 71.19; H, 8.64; S, 19.21\%. Elemental analytical data of P3HexTh have been discussed. ${ }^{14}$

Other polymers were prepared analogously. As described later, $\mathrm{CH}_{3} \mathrm{CN}$ as the solvent gave P3HexThs with relatively small PDI. Obtained polymers did not seem to contain the polymerization solvent because the content of nitrogen was negligible (within an experimental error): for example, analytical data of Run 14 P3HexTh in Table 1: Found: C, 71.65; H, 8.72; N, 0.09 (negligible, within experimental error); S, $18.62 \%$.

The HT content was estimated from $\alpha-\mathrm{CH}_{2}$ proton peaks observed at $\delta 2.80$ (HT unit) and $\delta 2.55$ (HH unit). HT content $=$ (peak area of the peak at $\delta$ $2.80) /\left(\right.$ peak area of the peak at $\delta 2.80+$ peak area of the peak at $\delta 2.55$ ). ${ }^{6}$

\section{RESULTS AND DISCUSSION}

Preparation of $\mathrm{P} 3 \mathrm{HexTh}$ was carried out under conditions shown in equation (3) and the results of the polymerization in hexane, $\mathrm{CHCl}_{3}$, $\mathrm{CH}_{2} \mathrm{Cl}_{2}$, and $\mathrm{CH}_{3} \mathrm{CN}$ are summarized in Table 1 .<smiles>c1cc(CCCCCc2ccsc2)cs1</smiles>

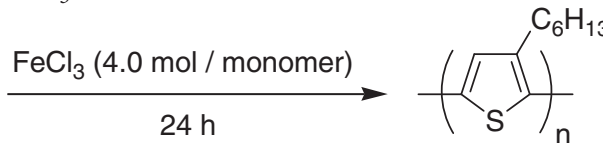

Hexane gave P3HexTh with low HT contents of $62 \%$ and $69 \%$ at room temperature and $-20^{\circ} \mathrm{C}$, respectively (Runs 1 and 2). Results of COP in $\mathrm{CHCl}_{3}$ (Runs 3-6) basically agreed with those reported previously. ${ }^{11} 3$-Hexylthiophene was also smoothly polymerized in $\mathrm{CH}_{2} \mathrm{Cl}_{2}$ and $\mathrm{CH}_{3} \mathrm{CN}$ to afford P3HexTh with high $\mathrm{HT}$ contents of
Table 1 Chemical oxidation polymerization of 3-hexylthiophene

\begin{tabular}{|c|c|c|c|c|c|c|c|}
\hline Run & Solvent & $\begin{array}{c}\text { Temperature } \\
\left({ }^{\circ} \mathrm{C}\right)\end{array}$ & $\begin{array}{l}\text { Yield } \\
(\%)\end{array}$ & $\begin{array}{l}\mathrm{M}_{n}^{\mathrm{a} x} \\
10^{-3}\end{array}$ & $P D I^{\mathrm{b}}$ & $\begin{array}{l}H T^{\mathrm{C}} \\
(\%)\end{array}$ & $\begin{array}{c}H T / H H^{c} \\
\text { ratio }\end{array}$ \\
\hline 1 & hexane & $\mathrm{rt}^{\mathrm{d}}$ & 65 & 18 & 3.3 & 62 & 1.6 \\
\hline 2 & hexane & -20 & 50 & 28 & 4.6 & 69 & 2.2 \\
\hline $\begin{array}{l}3 \\
(\mathrm{P} 1)\end{array}$ & $\mathrm{CHCl}_{3}$ & $\mathrm{rt}$ & 70 & 22 & 5.7 & 67 & 2.0 \\
\hline $\begin{array}{l}4 \\
(\mathrm{P} 2)\end{array}$ & $\mathrm{CHCl}_{3}$ & -20 & 70 & 19 & 2.8 & 81 & 4.3 \\
\hline $\begin{array}{l}5 \\
\text { (P3) }\end{array}$ & $\mathrm{CHCl}_{3}$ & -40 & 80 & 19 & 2.7 & 87 & 6.7 \\
\hline 6 & $\mathrm{CHCl}_{3}$ & -60 & 74 & 19 & 2.6 & 87 & 6.7 \\
\hline 7 & $\mathrm{CH}_{2} \mathrm{Cl}_{2}$ & rt & 67 & 34 & 4.0 & 78 & 3.4 \\
\hline 8 & $\mathrm{CH}_{2} \mathrm{Cl}_{2}$ & -20 & 55 & 31 & 4.1 & 83 & 4.9 \\
\hline 9 & $\mathrm{CH}_{2} \mathrm{Cl}_{2}$ & -40 & 50 & 46 & 5.0 & 87 & 6.7 \\
\hline 10 & $\mathrm{CH}_{2} \mathrm{Cl}_{2}$ & -60 & 45 & 46 & 4.3 & 87 & 6.7 \\
\hline 11 & $\mathrm{CH}_{3} \mathrm{CN}$ & $\mathrm{rt}$ & 70 & 34 & 1.5 & 75 & 3.0 \\
\hline 12 & $\mathrm{CH}_{3} \mathrm{CN}$ & 0 & 65 & 41 & 1.7 & 77 & 3.3 \\
\hline 13 & $\mathrm{CH}_{3} \mathrm{CN}$ & -20 & 54 & 45 & 1.7 & 83 & 4.9 \\
\hline 14 & $\mathrm{CH}_{3} \mathrm{CN}$ & -40 & 50 & 42 & 1.6 & 83 & 4.9 \\
\hline
\end{tabular}

aDetermined by gel-permeation chromatography (polystyrene standards, eluent $=$ chloroform) bolydispersity. $\mathrm{PDI}=M_{\mathrm{w}} / M_{\mathrm{n}}$

${ }^{\mathrm{CHT}}=$ content of the head-to-tail unit. ${ }^{6} \mathrm{HH}=$ content of the head-to-head unit ${ }^{d} \mathrm{rt}=$ room temperature $\left(\right.$ ca. $\left.25^{\circ} \mathrm{C}\right)$

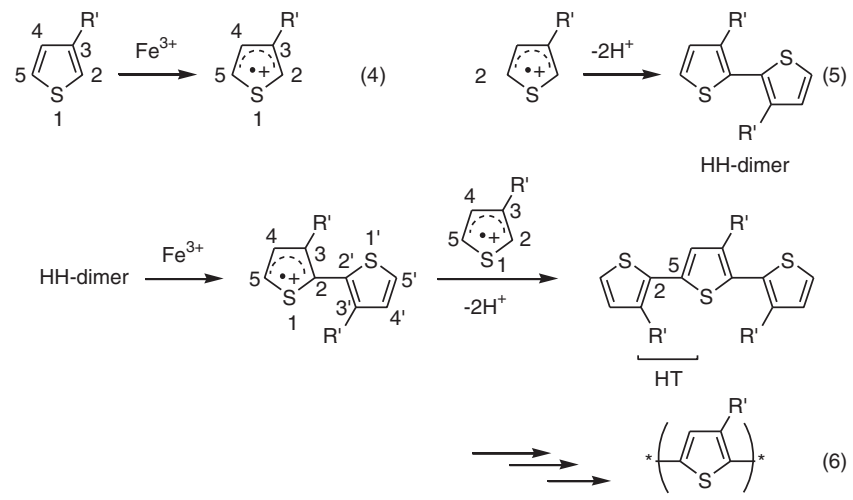

Scheme 1 Suggested mechanism for COP of 3-substituted thiophene. ${ }^{12}$

87\% (Runs 9 and 10) and 83\% (Runs 13 and 14), respectively. Results shown in Table 1 indicate that $\mathrm{COP}$ in $\mathrm{CH}_{2} \mathrm{Cl}_{2}$ and $\mathrm{CH}_{3} \mathrm{CN}$ gave P3HexTh with larger $M_{\mathrm{n}}$ than COP in $\mathrm{CHCl}_{3}$, and $M_{\mathrm{n}}$ became larger than 40000 at lower temperatures. In the case of $\mathrm{CH}_{3} \mathrm{CN}, \mathrm{P} 3 \mathrm{HexTh}$ had relatively narrow PDIs $\left(\mathrm{PDI}=M_{\mathrm{w}} / M_{\mathrm{n}}\right)$ of 1.5-1.7.

Barbarella et al. ${ }^{12}$ suggested the mechanism shown in Scheme $1\left(\mathrm{R}^{\prime}\right.$ in Scheme $1=-\mathrm{SR}$ in their paper) for COP of 3-substituted thiophene. First, two oxidized thiophene monomers (equation (4)) are thought to couple together at the 2-position to give head-to-head type bithiophene (HH-dimer) (equation (5)). ${ }^{11,12}$ As shown in equation (6), the 5-position of the oxidized $\mathrm{HH}$-dimer is considered to couple with the 2-position of the oxidized monomer to form a new HT bond in the trimer; the coupling reaction is accompanied by liberation of two $\mathrm{H}^{+}$. Repetition of such reaction is thought to finally give P3RTh with a high HT content.

As shown in Table 1, the HT content of P3HexTh increases by lowering polymerization temperature, revealing that formation of the HT unit is energetically advantageous than that of the $\mathrm{HH}$ unit. By 
lowering temperature from room temperature $\left(\mathrm{ca} .25^{\circ} \mathrm{C}\right)$ to $-40{ }^{\circ} \mathrm{C}$, the $\mathrm{HT} / \mathrm{HH}$ ratio becomes about double, which corresponds to Arrhenius' activation energy difference of about $6 \mathrm{~kJ} \mathrm{~mol}^{-1}$ between the HT-forming reaction and $\mathrm{HH}$-forming reaction. Regio-controlled HT-P3HexTh forms aggregates by self-assembly especially at low temperatures, ${ }^{3}$ and formation of stable aggregates of regio-controlled P3HexTh is thought to contribute to increase the HT content at low temperatures.

As shown in Table 1, choice of $\mathrm{CH}_{3} \mathrm{CN}$ gives HT-type P3HexTh with a relatively narrow molecular weight distribution (PDI $=1.5-$ 1.7), although $\mathrm{P} 3 \mathrm{HexTh}$ prepared by COP and OMP usually had a wider molecular weight distribution $(\mathrm{PDI}=\mathrm{ca} .2-4)$. Because of the narrower molecular weight distribution, the HT-type P3HexTh obtained in $\mathrm{CH}_{3} \mathrm{CN}$ may be used without fractionation. ${ }^{15}$

Effects of the HT content on physical and chemical properties of COP-prepared P3HexTh have been examined using P1-P3. P3 has a relatively high HT content and may show tendency to self-assemble similar to the case of OMP-prepared P3RTh, ${ }^{1-6}$ whereas P1 will show different physical and chemical properties from those of $\mathbf{P} \mathbf{3}$ because of its lower HT content. Table 2 summarizes UV-vis data and electrical conductivities of the polymers.

Figure 1 shows UV-vis spectra of P1-P3 films before doping and after p-doping. In $\mathrm{CHCl}_{3}$, the three polymers show only a UV-vis peak originated from single $\mathrm{P} 3 \mathrm{HexTh}$ molecules, giving rise to a peak in a range of $430-444 \mathrm{~nm}$ (cf. Table 2). ${ }^{3}$ In film, the UV-vis peak shifts to a longer wavelength, reflecting molecular assembly of the polymer in the solid state. The main peak of $\mathbf{P 1}$ in $\mathrm{CHCl}_{3}$ at $430 \mathrm{~nm}$ shifts to $477 \mathrm{~nm}$ in film (shift $(\Delta)=477-430=47 \mathrm{~nm}$ ), and P2 and P3 films give rise to main UV-vis peaks at $511 \mathrm{~nm}$ and $520 \mathrm{~nm}$ with larger $\Delta \mathrm{s}$ of $69 \mathrm{~nm}$ and $76 \mathrm{~nm}$, respectively. The larger shift of UV-vis peak of $\mathbf{P} 2$ and $\mathbf{P} 3$ by going from solution to film is thought to arise from $\pi$-stacking ${ }^{1-6}$ of $\mathbf{P} 2$ and $\mathbf{P} 3$ with larger HT content. The powder XRD data of P1-P3 shown in Figure 2 support this view, and $\mathbf{P} 3$ gives rise distinct XRD peaks at $d_{1}=15.9 \AA, d_{1} / 2=8.0 \AA, d_{1} / 3=5.3 \AA$ and $d_{2}=3.8 \AA$.

The XRD pattern of $\mathbf{P} 3$ essentially agrees with that of OMPprepared P3HexTh with a HT content of $98.5 \%,{ }^{3,6}$ and $d_{1}$ is assigned to a distance between $\mathrm{P} 3 \mathrm{HexTh}$ main chains separated by the hexyl side groups. ${ }^{3,6} d_{2}$ is thought to correspond to a $\pi$-stacking distance between the P3HexTh molecules. In contrast, P1 shows only a weak XRD peak at $d_{1}=16.1 \AA$, indicating that P1 does not form a wellpacked solid structure.

As shown in Table 2, P3 shows a higher electrical conductivity than $\mathbf{P} 1$ and $\mathbf{P} 2$ after oxidation (p-doping) with $\mathrm{NOBF}_{4} . \mathrm{NOBF}_{4}$ is a useful $\mathrm{p}$-doping (oxidizing) reagent for $\pi$-conjugated molecules and polymers. ${ }^{16,17}$ The polymer films cast on a Pt plate was dipped in a $\mathrm{CH}_{3} \mathrm{CN}$ solution of $\mathrm{NOBF}_{4}(0.1 \mathrm{M})$ for $15 \mathrm{~min}$ at room temperature. The $\mathrm{NOBF}_{4}$-doped film was peeled off from the plate, and electrical conductivity of the p-doped polymer film was measured with a resistometry by a four-probe method. All the $\mathrm{NOBF}_{4}$-oxidized ${ }^{16,17}$ polymer films show new peaks at about $700-800 \mathrm{~nm}$ and 1600 $2000 \mathrm{~nm}$ (cf. Figure 1b), which are assigned to a polaron and/or bipolaron band. By increasing the HT content, the new bands shift to a longer wavelength. P1 shows the new peaks at approximately $700 \mathrm{~nm}$ and $1600 \mathrm{~nm}$, whereas P2 and P3 give rise to the new peaks at approximately $800 \mathrm{~nm}$ and $2000 \mathrm{~nm}$ (Figure 1b). The difference is thought to essentially reflect the difference in the UVvis peaks between original non-doped $\mathbf{P 1}$ and $\mathbf{P} 2-\mathbf{P} 3$, and p-doped P2 and P3 may have a longer effective $\pi$-conjugation length and/or stronger intermolecular electronic interaction in solid than p-doped P1.
Table 2 UV-vis data and electrical conductivities of P1-P3

\begin{tabular}{cccc}
\hline & \multicolumn{2}{c}{ UV-Vis, $\lambda_{\max }(\mathrm{nm})$} \\
\cline { 2 - 3 } Polymer & Solution $^{\mathrm{a}}$ & Film $^{\mathrm{b}}$ & \\
\hline P1 & 430 & $\sigma^{\mathrm{c}}\left(\mathrm{Scm}^{-1}\right)$ \\
P2 & 442 & $517^{\mathrm{m}}, 556^{\mathrm{sh}}, 596^{\mathrm{sh}}$ & 0.1 \\
P3 & 444 & $520^{\mathrm{m}}, 554^{\mathrm{m}}, 600^{\mathrm{sh}}$ & 1.0 \\
\hline
\end{tabular}

${ }^{a}$ In $\mathrm{CHCl}_{3}$.

${ }^{b}$ Cast film on a quartz plate. $\mathrm{m}=$ main peak. $\mathrm{sh}=$ shoulder peak.

${ }^{c}$ Electrical conductivity of $\mathrm{NOBF}_{4}$-oxidized polymer film. ${ }^{16}$
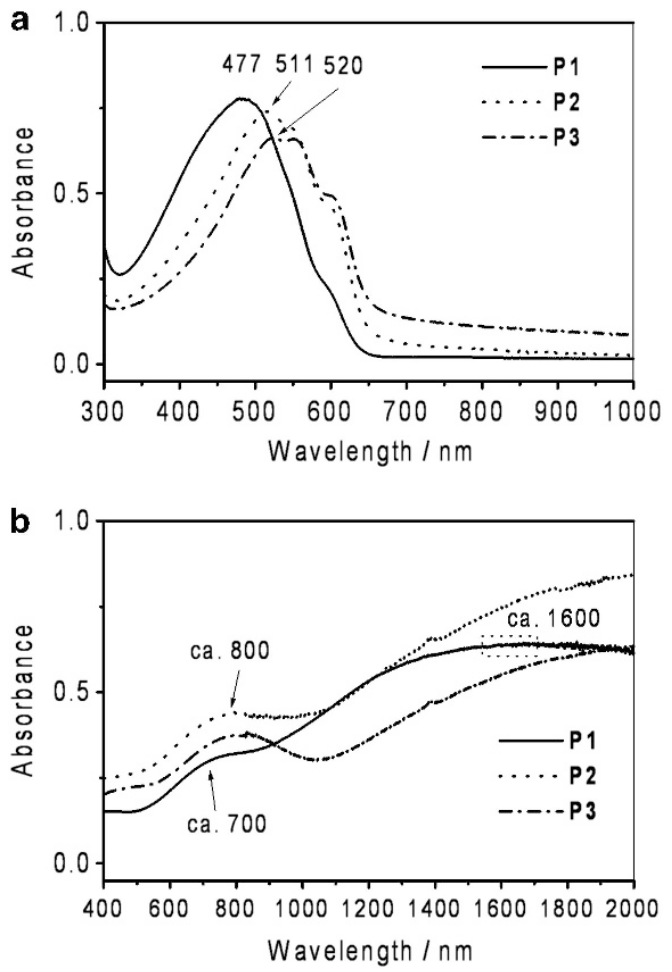

Figure 1 UV-vis spectra of P1-P3. (a) Non-doped and (b) NOBF 4 -oxdized films.

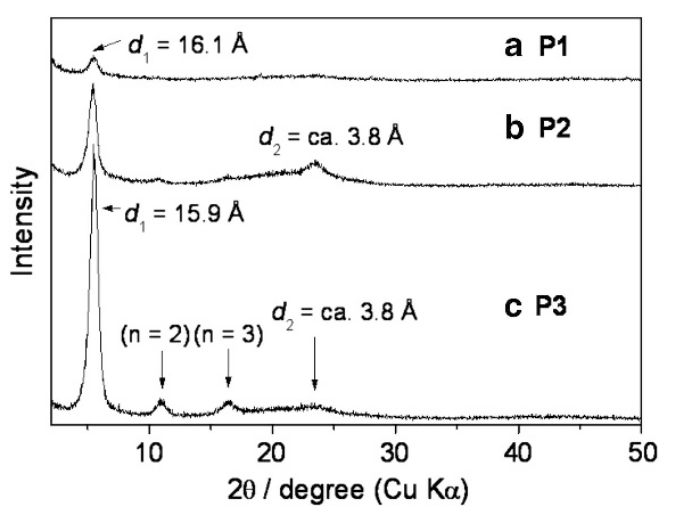

Figure 2 Powder XRD patterns of (a) P1, (b) P2 and (c) P3. 


\section{CONCLUSIONS}

Solvent and temperature effects of COP for the $\mathrm{P} 3 \mathrm{HexTh}$ preparation have been revealed, and effects of the HT content of the COPprepared $\mathrm{P} 3 \mathrm{HexTh}$ on its physical and chemical properties are reported. Acetonitrile gave $\mathrm{P} 3 \mathrm{HexTh}$ with a high molecular weight, a large HT content and a narrower PDI.

\section{ACKNOWLEDGEMENTS}

This work was partly supported by a Grant-in-Aid for Science Research in a Priority Area 'Super-Hierarchical Structures' from the Ministry of Education, Culture, Sports and Technology, Japan.

1 Sirringhaus, H., Brown, P. J., Friend, R. H., Nielsen, M. M., Bechgaard, K., LangeveldVoss, B. M. W., Spiering, A. J. H., Janssen, R. A. J., Meijer, E. W., Herwig, P. \& De Leeuw, D. M. Two-dimensional charge transport in self-organized, high-mobility conjugated polymers. Nature 401, 685-688 (1999).

2 Bao, Z. \& Lovinger, A. J. Soluble regioregular polythiophene derivatives as semiconducting materials for field-effect transistors. Chem. Mater. 11, 2607-2612 (1999).

3 Yamamoto, T., Komarudin, D., Arai, M., Lee, B. L., Suganuma, H., Asakawa, N., Inoue, Y., Kubota, K., Sasaki, S., Fukuda, T. \& Matsuda, H. Extensive studies on $\pi$-stacking of poly(3-alkylthiophene-2,5-diyl)s and poly(4-alkylthiazole-2,5-diyl)s by optical spectroscopy, NMR analysis, light scattering analysis, and X-ray crystallography. J. Am. Chem. Soc. 120, 2047-2058 (1998).

4 Yamamoto, T. Synthesis of $\pi$-conjugated polymers by organometallic polycondensation. Bull. Chem. Soc. Jpn. 83, 431-455 (2010).

5 McCullough, R. D., Tristam-Nagle, S., Lowe, S. P., Lowe, R. D. \& Jayaraman, M. Self-orienting head-to-tail poly(3-alkylthiophenes): new insights on structure-property relationships in conducting polymers. J. Am. Chem. Soc. 115, 4910-4911 (1993).

6 Chen, T., Wu, X. \& Rieke, R. D. Regiocontrolled synthesis of poly(3-alkylthiophenes) mediated by rieke zinc: their characterization and solid-state properties. J. Am. Chem. Soc. 117, 233-244 (1995).
7 Fukumoto, H., Kimura, R., Sasaki, S., Kubota, K. \& Yamamoto, T. Regioregular headto-tail poly(6-alkylpyridine-2,5-diyl)s and a new type of packing structure of the polymer in the solid state. J. Polym. Sci. B Polym. Phys. 43, 215-222 (2005).

8 Sugimoto, R., Takeda, S. \& Yoshino, K. Preparation of soluble polythiophene derivatives utilizing transition metal halides as catalysts and their property. Chem. Express 1, 635-638 (1986).

9 Shimizu, H., Yamada, M., Wada, R. \& Okabe, M. Preparation and characterization of water self-dispersible poly(3-hexylthiophene) particles. Polym. J. 40, 33-36 (2008).

10 Endo, K., Ogura, T., Higashihara, T. \& Ueda, M. A negative-type photosensitive poly (3-hexylthiophene) with cross-linker and photoacid generator. Polym. J. 41, 808-809 (2009).

11 Amou, S., Haba, O., Shirato, K., Hayakawa, T., Ueda, M., Takeuchi, K. \& Asai, M. Head-to-tail regioregularity of poly(3-hexylthiophene) in oxidative coupling polymerization with $\mathrm{FeCl}_{3}$. J. Polym. Sci. A Polym. Chem. 37, 1943-1948 (1999).

12 Barbarella, G., Zambianchi, M., Toro, R. D., Colonna, Jr. M., Iarossi, D., Goldoni, F. \& Bongini, A. Regioselective oligomerization of 3-(alkylsulfanyl)thiophenes with ferric chloride. J. Org. Chem. 61, 8285-8292 (1996).

13 Yu, S., Hayakawa, T. \& Ueda, M. Synthesis of poly(3-hexylthiophene) by using the $\mathrm{VO}(\mathrm{acac})_{2}-\mathrm{FeCl}_{3}-\mathrm{O}_{2}$ catalytic system. Chem. Lett. 559-560 (1999).

14 Yamamoto, T., Kokubo, H., Kondo, H., Akiyama, Y. \& Fujimura, I. Purification of headto-tail type regioregular poly(3-hexylthiophene), HT-P3RTh, and investigation of the effects of polymer purity on the performance of organic field-effect transistors. Jpn. J. Appl. Phys 42, 6627-6628 (2003).

15 Yamamoto, T., Honda, Y., Sato, T. \& Kokubo, H. Electrochemical behavior of poly (3-hexylthiophene). Controlling factors of electric current in electrochemical oxidation of poly(3-hexylthiophene)s in a solution. Polymer 45, 1735-1738 (2004).

16 Cao, J \& Curtis, M. D. Polarons, bipolarons, and $\pi$-dimers of bis(3,4-ethylenedioxythiophene)-(4,4'-dialkyl-2,2'-bithiazole)-co-oligomers. Direct measure of the intermolecular exciton transfer interaction. Chem. Mater. 15, 4424-4430 (2003).

17 Kumagai, A., Fukumoto, H. \& Yamamoto, T. Chemical and electrochemical oxidation of thiophene-pyridine and thiophene-pyrimidine co-oligomers in solutions. J. Phys. Chem. B 111, 8020-8026 (2007). 